\title{
Ductus arteriosus associated with an anomalous left coronary artery arising from the pulmonary artery: catastrophe after duct ligation
}

\author{
EDGARDO ORTIZ, MARC DE LEVAL, JANE SOMERVILLE \\ From the Paediatric and Adolescent Unit, National Heart Hospital, and the Hospital for Sick Children, Great \\ Ormond Street, London
}

SUMMARY Chronic left ventricular failure developed two months after ligation of an apparently uncomplicated large ductus arteriosus in a one year old girl. Two years later deterioration had progressed to a terminal stage. An anomalous left coronary artery arising from the pulmonary artery had not been recognised before duct ligation. This anomaly was diagnosed two years after ligation and was then treated by surgical redirection, but the patient died of severe left ventricular dysfunction.

The association of ductus arteriosus with an anomalous left coronary artery from the pulmonary artery has been reported once before. ${ }^{1}$ Recognition of the coronary anomaly before ligation of a large duct is difficult and the effect of routine ligation may be disastrous. We describe a patient with these associated lesions in whom the coronary abnormality was not diagnosed until two years after ligation. Despite surgical redirection of the anomalous artery she died.

\section{Case report}

A one year old girl from Oman was referred in chronic heart failure with signs of a large left to right shunt and collapsing pulses. She was a fullterm uncomplicated delivery (birth weight $3.5 \mathrm{~kg}$ ) and had developed heart failure at seven weeks. On admission she had signs of ductus arteriosus together with an apical pansystolic murmur suggestive of mitral regurgitation.

The chest $x$ ray showed cardiomegaly and plethora, and the electrocardiogram showed normal axis, $P$ mitrale, left ventricular hypertrophy with deep $S$ in V4R and V1, and deep but narrow $q$ waves preceding tall $R$ waves in V5 and V6 with normal upright $T$ waves.

Requests for reprints to Dr Jane Somerville, Paediatric and Adolescent Unit, National Heart Hospital, Westmoreland Street, London W1M 8BA.
Cross sectional echocardiography confirmed the large duct, a dilated but well contracting left ventricle, enlarged left atrium, a bicuspid aortic valve, and abnormal movement of the posterior mitral valve leaflet, which was attributed to papillary muscle dysfunction and ventricular dilatation.

The duct was ligated and her recovery was uneventful in the first week. A late systolic murmur persisted at the apex. The postoperative echocardiogram confirmed the same posterior mitral leaflet abnormality. The left ventricular cavity was smaller than before ligation; no comment was made on its function.

She returned to Oman on the eighth postoperative day. There was no further information until the consultant paediatrician sought the opinion of JS two years later. Apparently, the child had become unwell two months after her return home and since then she had suffered from congestive heart failure.

She had features of left-sided heart failure and chronic collapse of the left lung due to pressure on the left main bronchus from cardiomegaly. There were no murmurs, but the third heart sound was loud and the peripheral pulses were reduced. A possible explanation for the deterioration was the presence of an anomalous left coronary artery from the pulmonary artery. On advice she had been returned to the UK for investigation to exclude this.

On admission the electrocardiogram showed sinus rhythm, left axis deviation, smaller $\mathrm{qR}$ pattern with upright $T$ waves, smaller $R$ waves than before, and slightly depressed ST segments that were not diag- 


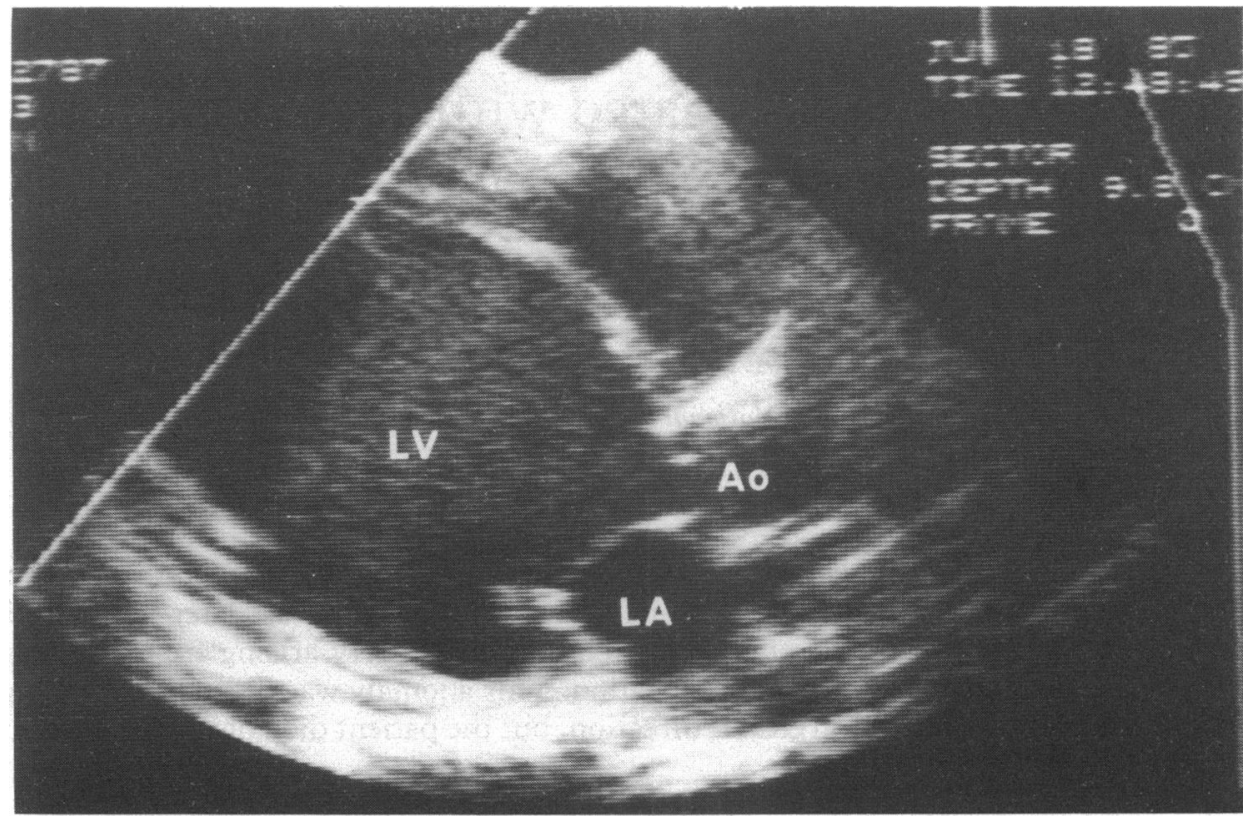

Fig. 1 Cross sectional echocardiogram in the parasternal long axis view at left sternal border showing the dilated left ventricular cavity and the thin interventricular septum and posterior wall of the left ventricle. Impaired ventricular function and poor movement of the mitral valve were demonstrated on real time. Ao, aorta; $L A$, left atrium; $L V$, left ventricle.

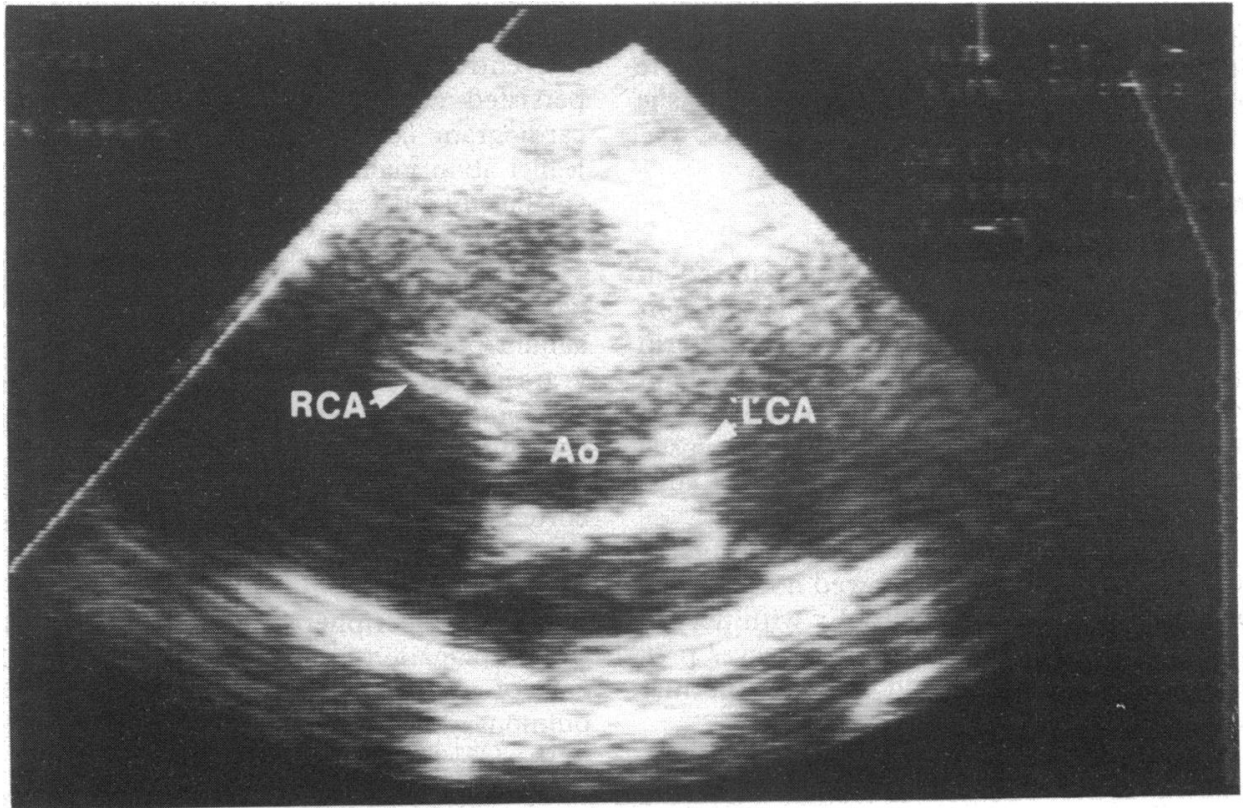

Fig. 2 Cross sectional echocardiogram in the parasternal short axis view showing the prominent right coronary artery and the "left" coronary artery originating from the aortic sinus. Ao, aorta; " $L$ " $C A$, "left" coronary atery; $R C A$, right coronary artery. 
nostic of infarction or ischaemia. Cross sectional echocardiography showed a dilated thin walled and poorly contracting left ventricle; these features were particularly pronounced in the anterior portion. The left atrium was enlarged and there was poor but symmetrical movement of the mitral cusps (Fig. 1) The coronary arteries appeared to emerge normally from the aorta (Fig. 2).

Cardiac catheterisation confirmed poor left ventricular function, with an ejection fraction of about $10 \%$ and end diastolic pressure of $20 \mathrm{~mm} \mathrm{Hg}$; the aortogram showed the large right coronary artery retrogradely perfusing the left coronary artery that entered the pulmonary artery posteriorly; the left anterior descending branch was not clearly seen.

Despite the high risk, because of poor ventricular function, we hoped that ligation or reimplantation of the left coronary artery might improve left ventricular function. After cardiac catheterisation she was intubated and ventilated, and treated with inotropes for 48 hours before operation. We had planned to ligate the left coronary artery but at operation we found two coronary orifices in the posterior sinus of the pulmonary artery which made this difficult. With cardiopulmonary bypass and cooling the left coronary orifices were directed to the aorta by means of a created aortopulmonary window and pericardial tunnel. The patient had difficulty coming off the bypass and when this was finally achieved the heart could not maintain coronary flow in diastole.

\section{Discussion}

When the left coronary artery originates from the pulmonary artery, the left ventricle is perfused with venous blood. Unless there is an increase in pulmonary diastolic pressure, there can be little or no perfusion from the pulmonary artery. In the fetus with an open duct and raised pulmonary vascular resistance, perfusion of the left ventricle is adequate for normal development. In the postnatal period, when the pulmonary vascular resistance falls and the duct closes, the perfusion of the left ventricle suffers and the effects of this are manifested weeks, months, and sometimes years later as ischaemic cardiomyopathy, cardiac infarction, left ventricular aneurysm, or mitral regurgitation.

If a large duct persists with hyperkinetic pulmonary hypertension, the left coronary artery will be perfused with oxygenated blood. The adequacy of perfusion will depend upon the degree to which pulmonary vascular resistance is increased. In our patient this probably was not high since although left ventricular function was slightly compromised there was no major infarction. Perhaps impaired left ventricular function contributed to papillary muscle dysfunction and hence mitral regurgitation. Duct closure produced an acute change in pulmonary artery pressure, flow, and oxygen saturation to the left coronary artery. Indeed in some cases it could be a cause of sudden deterioration or cardiac arrest after duct ligation. There was no sudden deterioration in our patient, however, presumably because the child was one year old at duct ligation and intercoronary collaterals had already developed to maintain left ventricular myocardial perfusion. This was inadequate for myocardial performance during normal active life.

Retrospective review of the postoperative echocardiogram showed definite evidence of impaired left ventricular contraction seven days after duct ligation. Left heart failure developed two months later due to ischaemic myopathy. By the time the condition was recognised the extensive myocardial damage was irreversible. If attention had been given to the deterioration in left ventricular function in the first postoperative week, earlier operation for the anomalous left coronary artery might have prevented the damage.

What measures may be taken to avoid missing the rare diagnosis of an anomalous coronary artery in the presence of a sizeable duct for which ligation (closure) is indicated? Normally no invasive investigation is done in patients with a ductus arteriosus, and even when catheterisation is performed, aortography is unlikely to show the anomalous coronary artery anatomy. The clinical and electrocardiographic features that are suggestive of an atypical duct were not present in this patient. Perhaps the early development of congestive heart failure in a fullterm baby with a patent duct and presence of mitral regurgitation with no demonstrable cusp abnormality should have suggested something unusual. Certainly the demonstration of decreased left ventricular function after duct ligation should have prompted early reinvestigation and a search for a coronary abnormality.

We have found that echocardiography is unreliable for the diagnosis of an anomalous coronary artery in infants and children because of the narrow separation between the transverse sinus of the pericardium and the left coronary artery as they pass behind the pulmonary trunk, a finding confirmed by others. ${ }^{2}$ When the appearance of the origins of the coronary arteries is unusual, selective angiography is indicated.

EO is a Squibb Medical Research Systems Fellow.

\section{References}

1 Vlodaver Z, Neufeld HN, Edwards JE. Coronary arterial variations in the normal heart and in congenital heart disease. New York: Academic Press, 1975.

2 Robinson PJ, Sullivan ID, Kumpeng V, Anderson RH, Macartney FJ. Anomalous origin of the left coronary artery from the pulmonary trunk. Potential for false negative diagnosis with cross sectional echocardiography. Br Heart $\mathcal{f}$ 1984; 52: 272-7. 